\title{
A Protocol on Antimicrobial effectiveness of adjunctive disinfection techniques during root canal therapy in patients with pulp necrosis and apical periodontitis. A Systematic Review \& Meta-analysis
}

\section{Durre Sadaf ( $\nabla$ d.ahmad@qu.edu.sa )}

Qassim University College of Dentistry https://orcid.org/0000-0003-4504-6267

\section{Zubair Ahmad}

Qassim University College of Dentistry

Igho - Onakpoya

University of Oxford

\section{Protocol}

Keywords: systematic review, randomized controlled trial, root canal therapy, disinfection, photodynamic therapy, ultrasonic irrigation, negative pressure technique, apical periodontitis

Posted Date: December 20th, 2019

DOl: https://doi.org/10.21203/rs.2.19281/v1

License: (c) (i) This work is licensed under a Creative Commons Attribution 4.0 International License.

Read Full License 


\section{Abstract}

Background The primary objective of root canal therapy is to eliminate bacteria from the root canal system of teeth. However, standard chemo-mechanical disinfection has been found unable to remove bacteria within the root canals. Clinicians tempted to use additional disinfection techniques to achieve adequate level of disinfection. There is no such systematic review that could comprehensively compare the efficiency of all currently used disinfection techniques. This systematic review aims to compare the antimicrobial effectiveness of various adjunctive disinfection techniques with standards chemomechanical disinfection technique(needle syringe irrigation) used during root canal therapy in patients with pulp necrosis and apical periodontitis.

Methods A well-defined structured clinical question in the form of PICO (Patients/Problem, Intervention, Comparison, Outcome) is designed. The unit of analysis in this review is a tooth. Teeth with apical periodontitis is a problem $(\mathrm{P})$. Interventions $(\mathrm{I})$ are supplemental disinfection techniques that include photodynamic therapy, passive ultrasonic irrigation, and negative pressure techniques. Comparison (C) will be standard chemo-mechanical disinfection technique. Outcome (0) is the effectiveness of antimicrobial activity in the form of detection of bacteria and colony-forming units (CFU). Keywords, $\mathrm{MeSH}$ terms, and synonyms will be searched through previous literature, books, and Medline database. We will perform a comprehensive search strategy using electronic databases MEDLINE (Ovid), EMBASE (Ovid), and Cochrane library. Hand searching of top endodontic journals and reference lists of selected studies will be carried out. Selection of studies will be made under predefined inclusion and exclusion criteria. Data extraction of selected studies will be done with two reviewers using a structured data collection form. At the same time, the risk of bias using the Cochrane risk of bias assessment tools will be carried out. A qualitative and quantitative data synthesis will be done. The data will be presented in the form of tables of summary of findings.

Discussion All potential clinical, methodological, and patient's related factors responsible for heterogeneity will be explored and discussed. We will also compare the results of our systematic review with existing literature. Strength and limitations of the systematic review will be discussed.Systematic Review registration Our protocol is registered with the International Prospective Register of Systematic Reviews (PROSPERO-CRD\#42019099141).

\section{Background}

Endodontic therapy or root canal therapy deals with pulpal and peri-radicular diseases. The leading cause of pulpal and periapical tissue diseases are bacteria. Therefore, complete elimination of bacteria or reducing bacteria at a substantial threshold level in the root canal system is the objective of root canal therapy $(1,2)$. The failure of root canal therapy is considered to be associated with bacteria left behind during the treatment, and it presents as persistence or emergence of apical periodontitis(3-5). Cultivable bacteria were found to be present in $30-50 \%$ of the root canals(6-8). 
Studies on the success and failure of root canal therapy showed that those cases in which bacteria culture after chemo-mechanical preparation was negative, the success rate was $94 \%$ than those cases with positive bacterial culture, the success rate was $64 \%(9)$. Other studies also reported the failure of healing of periapical radiolucency in the presence of persistent infection(9-11) in the root canals.

The standard chemo-mechanical disinfection technique is involved in removing infected pulpal tissues and dentin, shaping the root canals, and copious irrigation with sodium hypochlorite $(\mathrm{NaOCl})$ with the help of needle syringe technique. However, the standard chemo-mechanical disinfection of root canals is not able to eliminate bacteria from the root canal system(12). Multispecies bacterial biofilm within the complex root canal system is the main challenge and resistant to conventional chemo-mechanical disinfection technique(13). Conventional needle syringe irrigation has a drawback of being unable to irrigate apical third of the root canal system and unable to penetrate through dentinal tubules. One of the reasons is due to the vapor lock effect that hinders the irrigation solution to reach the apical parts of the root canal system(14). Therefore, adjunctive or supplemental disinfection techniques, i.e., negative pressure, sonic, passive ultrasonic, and use of laser and ozone gas, are recommended to overcome these challenges. Apical negative pressure technique is an alternate technique of irrigation, which is thought to decrease the risk of extrusion of irrigation solution through the apical foramen(15). It is expected to have greater efficacy in cleaning, debridement, and disinfection of the root canal system due to the lack of formation of vapor lock $(16,17)$.

Passive ultrasonic is also another adjunctive technique of irrigation that produces acoustic flow with forces and physically breaks the aggregates of bacterial colonies $(18,19)$. There are conflicting results on its antibacterial effects in different studies. A few systematic reviews on ultrasonic, the antimicrobial effect has been observed in in vitro studies $(20,21)$, while this systematic review will analyze the antimicrobial effects of passive ultrasonic in vivo studies.

Photodynamic therapy is another supplemental disinfection technique that involves the use of a photosensitizer followed by light illumination that converts this photosensitizer into toxic antibacterial species that leads to bactericidal effect(22).

There is no such systematic review to compare all supplemental techniques. Though there are several systematic reviews on specific technique $(21,23-25)$, there is a dearth of research on comparative evaluation of antimicrobial efficacy of these techniques.

This systematic review will assess the antibacterial effectiveness of the adjunctive disinfection technique and will help in evaluating comparative antibacterial efficacy.

\section{Methods}

This systematic review will be conducted under the guidance of Preferred Reporting Items for Systematic Review and Meta-Analysis (PRISMA-S)(26) and the Cochrane Handbook for Interventional Reviews. Our 
protocol is registered with the International Prospective Register of Systematic Reviews (PROSPEROCRD\#42019099141).

Review Question: What is the most effective adjunctive irrigation or disinfection technique in decreasing bacterial load (antimicrobial effect) from the root canal system during primary root canal therapy in patients with apical periodontitis compared to standard needle syringe irrigation method?

\section{Types of Studies}

This systematic review will include two kinds of study designs: one, randomized controlled trial with parallel groups and other Before-and-after study design in which microbial sample protocol S1, S2 and S3 is followed. In this protocol, the bacterial sample is taken before access preparation(S1), after chemomechanical preparation (S2), and after the use of adjunctive disinfection technique (S3).

\section{Types of Participants}

Adult patients who are diagnosed clinically and radiographically as pulp necrosis/apical periodontitis will be included. It will include both symptomatic and asymptomatic apical periodontitis. The review is limited to primary root canal therapy. However, there is no restriction to types of tooth; all kinds of single-rooted or multi-rooted teeth will be included.

Immature teeth, primary teeth, and previously root treated teeth with secondary infection will be excluded.

\section{Intervention}

The intervention is supplemental or adjunctive disinfectant techniques, i.e., passive sonic and ultrasonic irrigation, apical negative pressure, photodynamic therapy, ozone gas.

\section{Comparator}

Standard chemo-mechanical techniques, which are involved in debridement with rotary and hand instrumentation followed by irrigation with sodium hypochlorite with the help of a needle syringe technique, will be comparator.

\section{Outcome}

\section{Primary Outcome}

Data on the antimicrobial efficacy of the adjunctive disinfection technique will be collected in studies following S1, S2, and S3 protocol. However, data on bacterial load evaluation after further application of any intracanal medicament (S4) will not be extracted.

Antimicrobial efficacy evaluated by bacterial culture technique or polymerase chain reaction (PCR) will be measured in terms of: 
- Binary outcome as a negative and positive bacterial culture

- continuous outcome in the form of mean, range, and standard deviation of colony-forming units count (CFU). Both kinds of data binary and continuous (whichever is available) will be collected.

- Bacterial reduction in percentage.

\section{Secondary Outcome}

Other relevant secondary outcomes will be taken into count.

\section{Data sources and search strategies}

Electronic databases MEDLINE (Ovid), EMBASE (Ovid), and Cochrane library will be used from their inception to September 2019. There will be no restriction on language to any database.

We will also search for the clinical trial registry: http://clinicaltrial.giv. Hand searching of references for searched studies, existing systematic reviews, and endodontic journals will be carried out. We will also search

Two reviewers (SD, ZA) will work on the study selection process according to prespecified inclusion and exclusion criteria. Studies selected from all databases will be transferred to Endnote reference manager software (X8), where duplication of studies will be identified and removed. Initial screening of studies will be done on the basis of title and abstract. Then full-text articles of studies will be retrieved. The whole process of study selection will be presented as the PRISMA flow diagram(26).

The data extraction will be performed on a structured Microsoft Word \& Excel sheet. Details of study characteristics that include patient/problem, characteristics of the intervention, comparison, and outcome measures will be documented. One reviewer (DS) will extract data and will be crossed checked by the other reviewer $(\mathrm{ZA})$.

Authors of included studies will be contacted where necessary information is needed.

\section{Language}

Any language

\section{Risk of Bias Assessment}

The Cochrane Risk of Bias assessment tool will be used for assessing the risk of bias in the included studies. The studies will be classified as low, high, and unclear risk of bias based on the following six domains:

Random sequence generation: This is related to how studies generate a random sequence for equal distribution of patients in experimental and control groups. Online randomizers, coin tossing, and random number tables are considered an acceptable way of randomization. 
Allocation concealment: This domain determines whether the allocation of patients to various groups was adequately concealed from patients and operators. Studies that performed central allocation, sequentially numbered opaque sealed numbers are at low risk of bias while those used open number random allocation, date of birth, rotation will be considered at high risk of bias.

Blinding of participants, operators, and outcome assessors: Blinding is related to performance and detection bias. Studies, where lack of blinding or blinding of crucial personnel did not affect outcomes measures, will be considered at low risk of bias. However, studies where lack of blinding or incomplete blinding was found to affect outcomes, will be rank as high risk of bias.

Incomplete outcome data: If studies with no missing data or missing data are compensated across intervention groups, it will be considered at low risk. A high attrition rate and lack of intention to treat analysis will be considered at a high risk of bias.

Selective reporting bias: The included studies will be assessed for the reporting of all relevant outcome measures. Any discrepancy will be considered at a high risk of bias.

Overall quality of each study will be considered as "low" risk of bias when all the domains scored low, "some concerns" when at least one domain was scored as some concern and "high" when at least 1 domain scored as high or more than two domains as some concern.

Any disagreement between two reviewers (DS and ZA) will be resolved by discussion or by consulting the third reviewer (IP).

\section{Data synthesis}

The unit of analysis in this review is a tooth. The antibacterial efficacy is presented in the form of both continuous and dichotomous outcomes. We will collect both kinds of data available. For a dichotomous outcome, we will measure the risk ratio with $95 \%$ confidence interval. Continuous outcomes will be presented as weighted mean difference (WMD) with $95 \% \mathrm{Cl}$ or Standardized mean difference (SMD) with $95 \% \mathrm{Cl}$ in case if different measuring scales are used. The pooled estimate of the treatment effect will presented by using an appropriate model.

If sufficient homogeneity in terms of methodology and interventions among studies is found, metaanalysis using a fixed effect or random effect model will be conducted. Tests of heterogeneity (Chisquare) and $\mathrm{I}^{2}$ statistic will be conducted. If substantial heterogeneity is found ( $12>75 \%$, or $\left.\mathrm{P}<0.1\right)$, it may not be possible to conduct a meta-analysis, then a qualitative narrative summary of findings will be presented. The potential sources of heterogeneity will be explored by the methods of subgroup analysis and meta-regression.

Quality of evidence will be judged using the Grading of Recommendations, Assessment, Development, and Evaluation (GRADE) approach. Quality of evidence for each relevant outcome will be rated as high, moderate, low, and very low. 
Publication bias in the included studies will be assessed by a funnel plot.

\section{Discussion}

The purpose of this systematic review is to give a comprehensive and comparative analysis on different supplemental disinfection techniques used during root canal therapy and their antimicrobial effectiveness compared to standard chemo-mechanical disinfection technique. The main findings of the comparisons and size of effect will be presented in the form of tables of summary.

All potential clinical, methodological, and patient's related reasons for heterogeneity will be explored and discussed. We will also compare the results of our systematic review with existing literature. This review includes extensive literature search through electronic databases, explicit inclusion and exclusion criteria for studies selection by two independent reviewers, and assessment of risk of bias in included studies. Quality of evidence of the primary outcomes will be assessed by using GRADE that includes independent analysis of bias risk, accuracy, consistency, publication bias and indirect evidence. Heterogeneity in research design and methodologic variations may be limiting factors.

This systematic review has practical implication, and it will provide evidence-based information on additional disinfection achieved by supplemental techniques.

This review will also help in identifying areas of further research and hopefully inform future healthcare policy.

\section{References}

1. Haapasalo M, Endal U, Zandi H, Coil JM. Eradication of endodontic infection by instrumentation and irrigation solutions. Endodontic Topics. 2005;10(1):77-102.

2. Siqueira JF, Jr., Rocas IN. Clinical implications and microbiology of bacterial persistence after treatment procedures. Journal of endodontics. 2008;34(11):1291-301.e3.

3. Vieira AR, Siqueira JF, Jr., Ricucci D, Lopes WS. Dentinal tubule infection as the cause of recurrent disease and late endodontic treatment failure: a case report. Journal of endodontics. 2012;38(2):2504.

4. Signoretti FG, Endo MS, Gomes BP, Montagner F, Tosello FB, Jacinto RC. Persistent extraradicular infection in root-filled asymptomatic human tooth: scanning electron microscopic analysis and microbial investigation after apical microsurgery. Journal of endodontics. 2011;37(12):1696-700.

5. Fabricius L, Dahlen G, Sundqvist G, Happonen RP, Moller AJ. Influence of residual bacteria on periapical tissue healing after chemo-mechanical treatment and root filling of experimentally infected monkey teeth. Eur J Oral Sci. 2006;114(4):278-85.

6. Xavier ACC, Martinho FC, Chung A, Oliveira LD, Jorge AOC, Valera MC, et al. One-Visit Versus TwoVisit Root Canal Treatment: Effectiveness in the Removal of Endotoxins and Cultivable Bacteria. Journal of endodontics. 2013;39(8):959-64. 
7. Martinho FC, Gomes APM, Fernandes AMM, Ferreira NS, Endo MS, Freitas LF, et al. Clinical Comparison of the Effectiveness of Single-file Reciprocating Systems and Rotary Systems for Removal of Endotoxins and Cultivable Bacteria from Primarily Infected Root Canals. Journal of endodontics. 2014;40(5):625-9.

8. Ferreira NS, Martinho FC, Cardoso FGR, Nascimento GG, Carvalho CAT, Valera MC. Microbiological Profile Resistant to Different Intracanal Medications in Primary Endodontic Infections. Journal of endodontics. 2015;41(6):824-30.

9. Sjogren U, Figdor D, Persson S, Sundqvist G. Influence of infection at the time of root filling on the outcome of endodontic treatment of teeth with apical periodontitis. International endodontic journal. 1997;30(5):297-306.

10. Bonsor SJ, Nichol R, Reid TMS, Pearson GJ. Microbiological evaluation of photo-activated disinfection in endodontics (An in vivo study). British dental journal. 2006;200(6):337-41.

11. Trope M, Delano EO, Ørstavik D. Endodontic treatment of teeth with apical periodontitis: Single vs. Multivisit treatment. Journal of endodontics. 1999;25(5):345-50.

12. Siqueira JF, Jr. Aetiology of root canal treatment failure: why well-treated teeth can fail. International endodontic journal. 2001;34(1):1-10.

13. Chavez de Paz LE. Redefining the persistent infection in root canals: possible role of biofilm communities. Journal of endodontics. 2007;33(6):652-62.

14. Tay FR, Gu L-s, Schoeffel GJ, Wimmer C, Susin L, Zhang K, et al. Effect of Vapor Lock on Root Canal Debridement by Using a Side-vented Needle for Positive-pressure Irrigant Delivery. Journal of endodontics. 2010;36(4):745-50.

15. Nielsen BA, Craig Baumgartner J. Comparison of the EndoVac system to needle irrigation of root canals. Journal of endodontics. 2007;33(5):611-5.

16. Siu C, Baumgartner JC. Comparison of the debridement efficacy of the EndoVac irrigation system and conventional needle root canal irrigation in vivo. Journal of endodontics. 2010;36(11):1782-5.

17. Parente JM, Loushine RJ, Susin L, Gu L, Looney SW, Weller RN, et al. Root canal debridement using manual dynamic agitation or the EndoVac for final irrigation in a closed system and an open system. International endodontic journal. 2010;43(11):1001-12.

18. Burleson A, Nusstein J, Reader A, Beck M. The in vivo evaluation of hand/rotary/ultrasound instrumentation in necrotic, human mandibular molars. Journal of endodontics. 2007;33(7):782-7.

19. van der Sluis LW, Versluis M, Wu MK, Wesselink PR. Passive ultrasonic irrigation of the root canal: a review of the literature. International endodontic journal. 2007;40(6):415-26.

20. Caputa PE, Retsas A, Kuijk L, Chavez de Paz LE, Boutsioukis C. Ultrasonic Irrigant Activation during Root Canal Treatment: A Systematic Review. Journal of endodontics. 2019;45(1):31-44.e13.

21. Nagendrababu V, Jayaraman J, Suresh A, Kalyanasundaram S, Neelakantan P. Effectiveness of ultrasonically activated irrigation on root canal disinfection: a systematic review of in vitro studies. Clinical oral investigations. 2018;22(2):655-70. 
22. Mohammadi Z. Chemo-mechanical strategies to manage endodontic infections. Dentistry today. 2010;29(2):91-2, 4, 6 passim; quiz 9.

23. Fransson $\mathrm{H}$, Larsson KM, Wolf E. Efficacy of lasers as an adjunct to chemo-mechanical disinfection of infected root canals: a systematic review. International endodontic journal. 2013;46(4):296-307.

24. Konstantinidi E, Psimma Z, Chavez de Paz LE, Boutsioukis C. Apical negative pressure irrigation versus syringe irrigation: a systematic review of cleaning and disinfection of the root canal system. International endodontic journal. 2017;50(11):1034-54.

25. Chrepa V, Kotsakis GA, Pagonis TC, Hargreaves KM. The effect of photodynamic therapy in root canal disinfection: a systematic review. Journal of endodontics. 2014;40(7):891-8.

26. Moher D, Liberati A, Tetzlaff J, Altman DG. Preferred reporting items for systematic reviews and meta-analyses: the PRISMA statement. PLoS medicine. 2009;6(7):e1000097.

\section{Amendments}

\section{Amendments:}

A few changes were made in the previous protocol. The title is changed from network meta-analysis to systematic review and meta-analysis. Inclusion and exclusion criteria were changed. This systematic review is limited to supplemental disinfection techniques, i.e., sonic, ultrasonic, negative pressure technique, photodynamic therapy and ozone gas. Different irrigation protocols and the use of intracanal medications were excluded now. Search strategy and data synthesis was also modified.

\section{Support}

No financial support, the review is self-funded.

\section{Systematic Review Registration}

Our protocol is registered with the International Prospective Register of Systematic Reviews (PROSPEROCRD\#42019099141).

\section{Supplementary Files}

This is a list of supplementary files associated with this preprint. Click to download.

- SearchstrategyonMedineviaOvid.docx

- PRISMAPchecklist.docx 